\title{
E3 ligase Hei10: a multifaceted structure-based signaling molecule with roles within and beyond meiosis
}

\author{
Arnaud De Muyt, ${ }^{1,2,5}$ Liangran Zhang, ${ }^{3,5}$ Tristan Piolot, ${ }^{4}$ Nancy Kleckner, ${ }^{3}$ Eric Espagne,, \\ and Denise Zickler ${ }^{1,6}$ \\ ${ }^{1}$ UMR 8621, Institut de Génétique et Microbiologie, Université Paris-Sud, 91405 Orsay, France; ${ }^{2}$ Institut Curie, 75248 Paris \\ Cedex 05, France; ${ }^{3}$ Department of Molecular and Cellular Biology, Harvard University, Cambridge, Massachusetts 02138, USA; \\ ${ }^{4}$ UMR 3215, INSERM U934, Institut Curie, 75005 Paris, France
}

\begin{abstract}
Human enhancer of invasion-10 (Hei10) mediates meiotic recombination and also plays roles in cell proliferation. Here we explore Hei10's roles throughout the sexual cycle of the fungus Sordaria with respect to localization and effects of null, RING-binding, and putative cyclin-binding (RXL) domain mutations. Hei10 makes three successive types of foci. Early foci form along synaptonemal complex (SC) central regions. At some of these positions, depending on its RING and RXL domains, Hei10 mediates development and turnover of two sequential types of recombination complexes, each demarked by characteristic amplified Hei10 foci. Integration with ultrastructural data for recombination nodules further reveals that recombination complexes differentiate into three types, one of which corresponds to crossover recombination events during or prior to SC formation. Finally, Hei10 positively and negatively modulates SUMO localization along SCs by its RING and RXL domains, respectively. The presented findings suggest that Hei10 integrates signals from the $\mathrm{SC}$, associated recombination complexes, and the cell cycle to mediate both the development and programmed turnover/evolution of recombination complexes via SUMOylation/ubiquitination. Analogous cell cycle-linked assembly/disassembly switching could underlie localization and roles for Hei10 in centrosome/spindle pole body dynamics and associated nuclear trafficking. We suggest that Hei10 is a unique type of structure-based signal transduction protein.
\end{abstract}

[Keywords: Hei10; meiosis; recombination; synaptonemal complex; spindle pole body/centrosome]

Supplemental material is available for this article.

Received February 20, 2014; revised version accepted April 16, 2014.

Human enhancer of invasion-10 (Hei10) belongs to the Zip3/Hei10 broad family of molecules that comprises two major subgroups: the Hei10/CCNB1IP1 family and the Zip3/RNF212 family (Chelysheva et al. 2012). Budding yeast and worms encode only a Zip3 family member (Agarwal and Roeder 2000; Jantsch et al. 2004; Bhalla et al. 2008), plants plus Sordariales encode only a Hei10 family member (Chelysheva et al. 2012; Wang et al. 2012), and mammals encode one member of each family (Toby et al. 2003; Ward et al. 2007; Strong and Schimenti 2010; Reynolds et al. 2013; Qiao et al. 2014).

Hei10 was originally identified as a growth regulatory gene (Toby et al. 2003). It is hyperexpressed in certain cancer cells (Singh et al. 2007) but paradoxically can also constrain cancer proliferation (Singh et al. 2007; Scoles

\footnotetext{
${ }^{5}$ These authors contributed equally to this work.

${ }^{6}$ Corresponding authors

E-mail denise.zickler@igmors.u-psud.fr

E-mail eric.espagne@igmors.u-psud.fr

Article is online at http://www.genesdev.org/cgi/doi/10.1101/gad.240408.114.
}

2008). Sequence inspection identifies determinants that specify E3 ligase activity and SUMO binding (and thus post-translational modification via ubiquitin/SUMO) and both cyclin binding and cyclin-dependent kinase (CDK) phosphorylation (and thus interplay with the cell cycle) (Supplemental Fig. 1). Correspondingly, mammalian Hei10 exhibits ubiquitin ligase activity (Toby et al. 2003), interacts with SUMO and SUMOylated proteins (Strong and Schimenti 2010), and modulates cell cycle progression at G2/M when expressed in budding yeast (Toby et al. 2003).

During meiosis, Hei10 marks the sites of crossovers (COs) in Arabidopsis, rice, and mice (Ward et al. 2007; Chelysheva et al. 2012; Wang et al. 2012; Qiao et al. 2014). Hei10 colocalizes with the meiosis-specific MutS homolog Msh4/5, an early recombination marker; the MutL homo- 
$\log$ Mlh1, which likely mediates the final step of CO recombination; and the cyclin-dependent kinase 2 (Ward et al. 2007; Chelysheva et al. 2012; Wang et al. 2012; Luo and al. 2013; Qiao et al. 2014). Recent data suggest that Hei10 and Zip3/RNF212 have antagonistic roles for Msh4 stabilization (Wang et al. 2012; Luo et al. 2013; Qiao et al. 2014).

The above information implicates Hei10 as a strong candidate for integration of multiple signal inputs. The need for such integration is particularly important for meiosis, where local events of recombination must be temporally and spatially coordinated with developing chromosome axes plus synaptonemal complex (SC) formation on a per-nucleus basis to promote synchronous progression of events from stage to stage at all recombination sites. To explore such roles, we identified the Heilo gene encoded by the filamentous fungus Sordaria macrospora, performed detailed cytological localization analysis of Heilo in wild-type and recombination plus SC mutants, and carried out functional analysis of defects conferred by hei10 mutations, including a complete deletion null allele and targeted mutations predicted to disrupt E3 ligase and cyclin-binding functions.

Our analysis provides a coherent view of the progression of CO-fated recombination through Heilo association with Msh4 and its relationship to SC formation. We defined SUMO localization and its relationship to and dependence on Heilo and its subfunctions. Mutant phenotypes suggest that Heilo is a structure-based signal transduction protein that integrates structure cues from the SC, local cues from underlying DNA recombination events, and the cell cycle to mediate the programmed assembly and disassembly of different types of recombination complexes. We propose that these effects are implemented by a ubiquitin/SUMO "switch." We also discovered localization and roles for Heilo in centrosome/spindle pole body (SPB) dynamics and likely associated nuclear trafficking. Taken together, our results provide new perspectives from which to consider roles of Hei10 outside of meiosis.

\section{Results}

Three types of Hei10 foci emerge sequentially downstream from SC installation

Localization of Hei10 during meiosis was monitored by fluorescent protein fusions, Hei10-GFP and Hei10mCherry (Supplemental Material). Heilo foci are first detected at zygotene along the forming SC central regions. (1) They occur only on already-formed SC, never in unsynapsed regions (Fig. 1A-C). (2) Costaining of Hei10 with the SC central component Zip4 (E Espagne and D Zickler, unpubl.) shows that Zip4 foci colocalize with Hei10 foci (Fig. 1D-F). (3) Colocalization with SC central component Sme4-GFP (Espagne et al. 2011) reveals that Heilo foci localize to initial sites of synapsis, mostly at the borders of short Sme4 lines (Fig. 1G; Supplemental Fig. S2A-C).

In accord with specific localization to SC central regions, formation of Heilo foci is strictly dependent on SC formation. Foci do not form in sme4D or zip4D mutants, where SC does not form (Supplemental Fig. S2D,E). In cohesin (rec8D) and recombination (spo11D, mer3D, and $m s h 4 D$ ) mutants where SC formation is abnormal, Hei10 patterns match synapsis patterns: Hei10 foci occur only in closely aligned and synapsed regions of rec8D (Fig. $1 \mathrm{H}$ ); are absent in mer3D, which lacks SC (Supplemental Fig. S2F,G); are restricted to partially synapsed regions in $m s h 4 D$ (Fig. $1 \mathrm{I}$ ); and are seen in $30 \%$ of spo $11 D$ nuclei, in nonhomologously synapsed regions (Supplemental Fig. S2H-J).

Temporal analysis of Hei10 foci formation identifies three successively appearing types of SC-associated Heil0 foci, called T1 to T3 (Fig. 1J). Correspondingly, Hei10 focus diameters exhibit a trimodal distribution, implying two binary steps in Heilo focus maturation (Supplemental Fig. S2K).

T1 foci emerge during early and mid-zygotene (Fig. 1E). They are small (188 $\pm 2 \mathrm{~nm}$ in diameter), show regular sizes and brightness (Fig. $1 \mathrm{~K}, \mathrm{~L}$ ), and are regularly spaced $(0.47-0.56 \mu \mathrm{m} ; n=200$ foci) (arrows in Fig. 1L, $\gamma$ analysis parameter $\boldsymbol{v}>100)$. When synapsis is completed, all homolog pairs are decorated with T1 foci (110-120 per nucleus; $n=50$ ) (Fig. 1J). T1 foci colocalize with Zip4, an SC central region component (Fig. 1D-F; E Espagne and D Zickler, unpubl.), along all homologs and stay like Zip4 foci until late pachytene (see below). T1 also colocalizes with foci of Mer3 helicase (Supplemental Fig. S2L-N), which mark sites of total recombination interactions (Storlazzi et al. 2010).

T2 and T3 foci come up sequentially from late zygotene through pachytene (Fig. 2A-J). Early, mid-, and late pachytene substages can be defined with accuracy by ascus/meiocyte size and chromatin compactness, thus permitting detailed temporal analysis (Supplemental Fig. S3A-I). At late zygotene/early pachytene, $\sim 40$ (40.1 \pm $8.3 ; n=100$ nuclei) obviously brighter and larger Heilo foci emerge along the homologs (diameter $243 \pm 9 \mathrm{~nm}$; $n=200$ foci). These "T2" foci (Fig. 2A-C) emerge within already-formed arrays of $\mathrm{T} 1$ foci, which occur continuously between $\mathrm{T} 2$ foci and retain their earlier morphology and spacing (Fig. 2A). Thus, T2 foci appear to evolve from a subset of already-formed $\mathrm{T} 1$ foci.

Next, at mid-pachytene, T2 foci progressively disappear (Fig. 2D) and are replaced by $\sim 22$ (22 plus or minus three; $n=120$ nuclei) larger (diameter $324 \pm 3 \mathrm{~nm} ; n=$ 200) and brighter T3 foci (Fig. 2E,F), which then slowly diminish in number during late pachytene (to $\sim 15-17 ; n=$ 100 nuclei) (Fig. 2I,J). As for T2 foci (Fig. 2A), regularly spaced T1 foci remain present between all T3 foci (Fig. 2E,I). T3 foci persist through the early-mid-diffuse stage, where they remain associated with a residual stretch of SC (Fig. 2K; Supplemental Fig. S3J-L), and during diplotene, where they remain associated with chiasmata sites (Fig. 2L; Supplemental Fig. S3M-O).

Three-dimensional structured illumination microscopy (3D-SIM) shows that T3 foci are indeed specifically localized between the two homologous axes and partially protrude above the plane of the SC (Fig. 2F-H). Interestingly, about half of the foci are located near sites of SC 

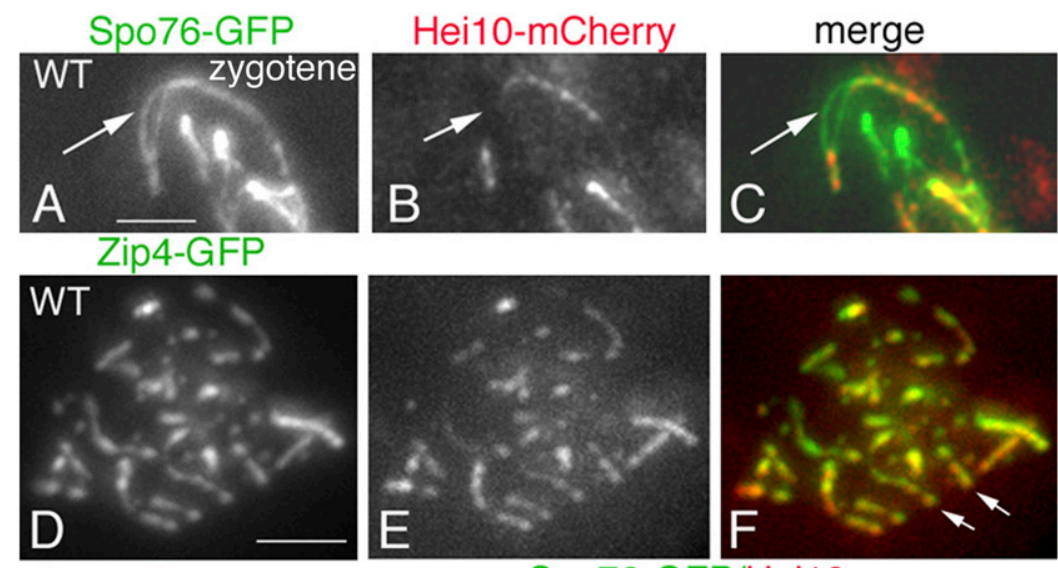

Sme4-GFP/Hei10
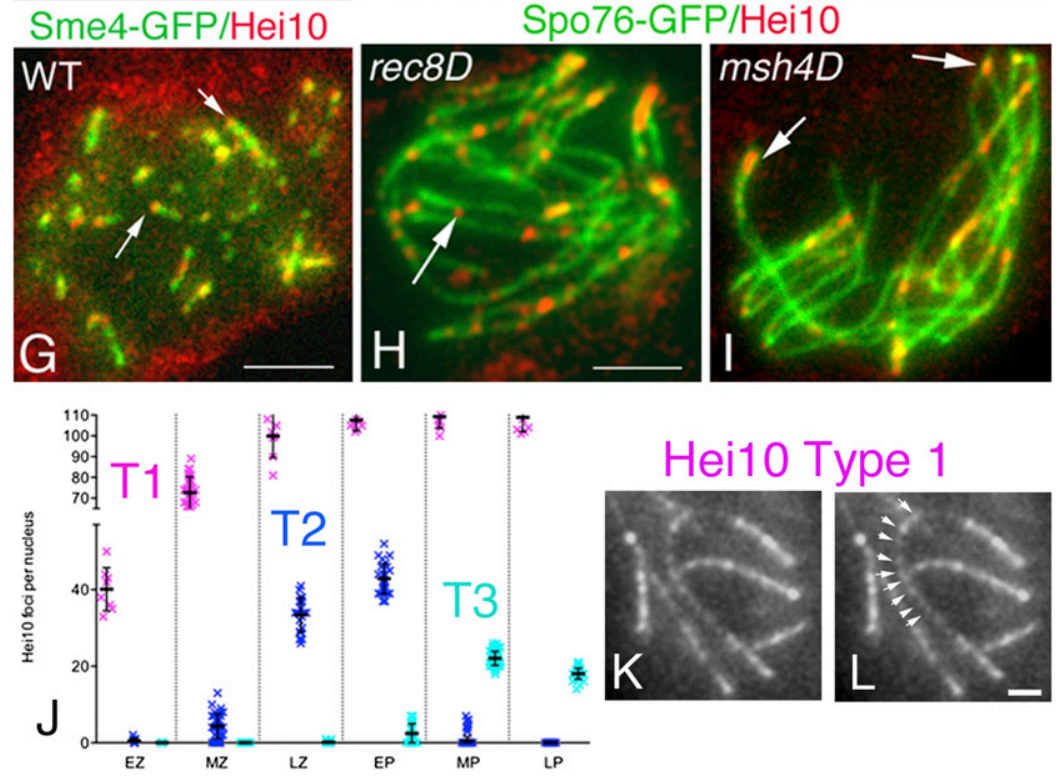

Figure 1. Heilo localization is SC-dependent. $(A-I)$ Heil0 is tagged by mCherry (Heil0); all nuclei are at zygotene. $(A-C)$ Costaining of Spo76-GFP $(A)$ with Heilo $(B)$. $(C)$ Merge. No Heilo foci are seen in the unsynapsed region (arrow). $(D-F)$ Zip4-GFP $(D)$ with Hei10 $(E)$. $(F)$ Merge. Hei10 foci colocalize with all Zip4 foci (arrows in merge). (G) Sme4-GFP with Heil0. Heil0 is often seen at ends of Sme4 segments (arrows) (details in Supplemental Fig. S2A-C); the "red" background is due to Heil0 perinuclear localization (see below). (H) Spo76-GFP with Heilo in rec $8 D$. The arrow points to a Heilo focus formed at a synapsing site. (I) Spo76-GFP with Heilo in msh4D. Only synapsed regions contain Heilo foci (arrows). ( $J$ ) Evolution of the three types of Hei10 foci (T1, T2, and T3) through prophase. (EZ) Early zygotene; (MZ) mid-zygotene; (LZ) late zygotene; (EP) early pachytene; (MP) mid-pachytene; (LP) late pachytene. See also Supplemental Figure S2L for the frequency distribution of their focus diameters. $(K, L) \mathrm{T} 1$ foci at mid-zygotene are uniform in brightness and size and are regularly spaced (arrows). Bars: $2 \mu \mathrm{m} ; L$, $400 \mathrm{~nm}$. twists (arrows in Fig. 2G). This structural distortion points to structural and/or geometric interplay between the DNA events of $\mathrm{CO}$ formation and the SC.

Type T2 and type T3 Hei10 foci are linked to maturation and turnover of decreasing subsets of Msh4 foci

The meiosis-specific MutS homolog Msh4 binds and stabilizes DNA strand exchange intermediates (Snowden et al. 2004). In Sordaria, Msh4 foci emerge on forming SCs at early zygotene and, by early pachytene, mark all recombination sites as defined by genetic and cytological analyses (81 plus or minus seven at early pachytene; $n=$ 100 nuclei). Their number then decreases to 50 plus or minus eight through mid-pachytene and then further decreases prior to complete disappearance at the mid-/late pachytene transition (Storlazzi et al. 2010). Thus, in contrast to Hei10, which shows three morphologically different types of foci (see above), Msh4 foci exhibit what appears to be a progressive diminution without any change in morphology. Nonetheless, covisualization of Msh4 and Heilo focus patterns in the same nuclei reveals a temporal program in which specific, decreasing subsets of Msh4 complexes colocalize with specific types of Heilo foci.

From late zygotene through early pachytene, all T2 foci colocalize with an Msh4 focus, whereas $\sim 40 \%$ of Msh4 foci do not have a colocalizing T2 focus and instead colocalize with T1 foci ( $n=200$ foci) (Fig. 3A-C). Thus, T2 foci occur at a subset of total Msh4 recombination interactions. From early pachytene (Fig. 3D-F) to midpachytene (Fig. 3G-I), after $\sim 40 \mathrm{~T} 2$ foci have formed, the total number of Msh4 foci diminishes from $\sim 80$ to $\sim 50$ (see above), suggesting that Msh4/T2 complexes are specifically retained, while non-T2-associated Msh4 foci are lost. It thus appears that $\mathrm{T} 2$ foci mediate the stabilization of Msh4 foci at a specific subset of $\sim 40$ recombinational interactions.

By mid-pachytene, in nuclei where $\mathrm{T} 2$ foci have disappeared and a smaller number of $\mathrm{T} 3$ foci have emerged, the number of Msh4 foci has further diminished, and almost all remaining Msh4 foci now colocalize with a T3 focus $(90 \% ; n=100)$ (Fig. 3J-L). Thus, among the $\sim 40$ previously T2/Msh4 cocomplexes, $\sim 22$ have evolved into Msh4/T3 cocomplexes, while the remaining $\sim 18$ have lost 


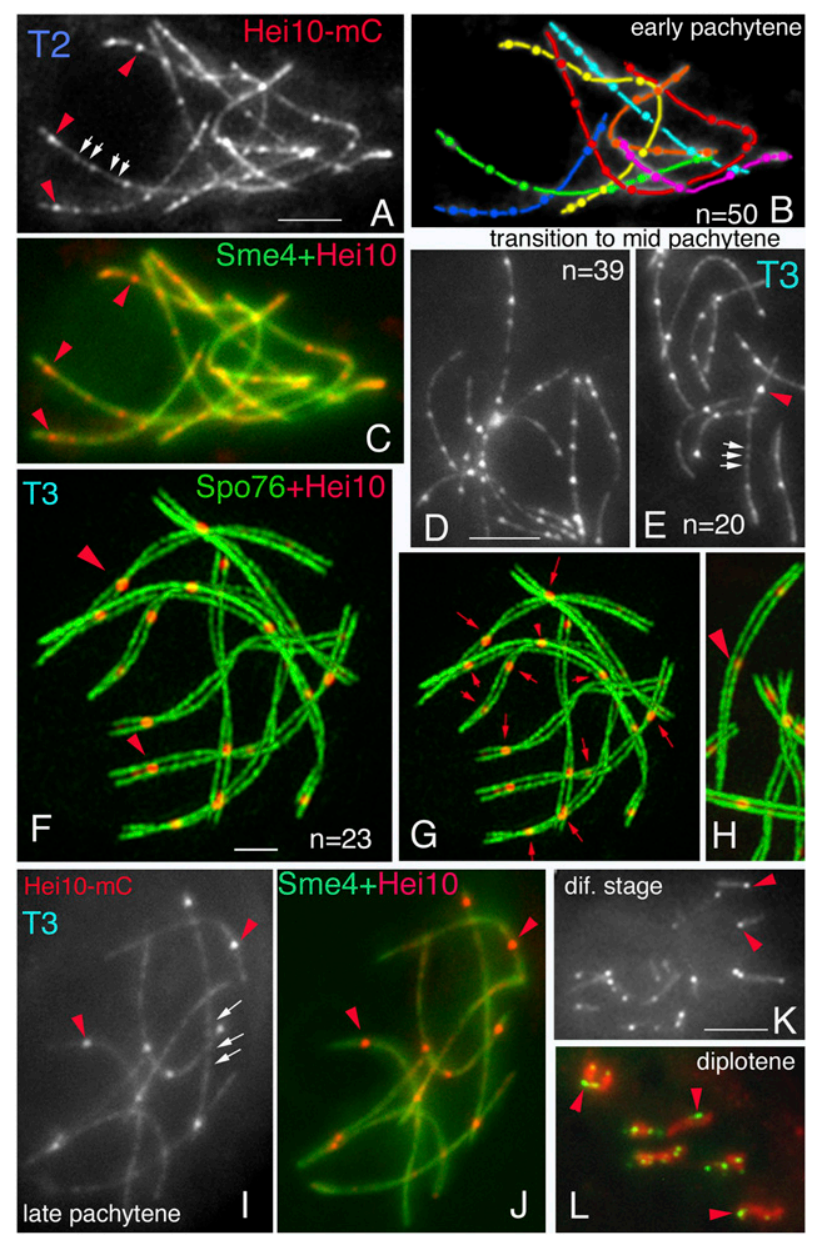

Figure 2. Evolution of $\mathrm{T} 2$ and $\mathrm{T} 3$ foci throughout prophase. $(A-C)$ Early pachytene. $(A) \mathrm{T} 1$ foci (white arrows) remain, but a subset of foci (red arrowheads, also in $C$ ) become larger and brighter. $(B)$ Cartoon of T2 foci along the seven bivalents. $(C)$ Merged picture of Heil0-mCherry with Sme4-GFP. $(D, E)$ Transition from early pachytene $(D)$ to mid-pachytene $(E)$. (E) T1 foci (white arrows) are still present, but T2 foci (red arrowhead) increase in brightness and progressively reduce in numbers. T1 foci are clear in some Z-sections $(E)$ but appear as faint lines in section overlays $(D, E)$. $(F-H)$ 3D-SIM picture of a mid-pachytene nucleus costained by Spo76-GFP and Heil0-mCherry. $(F)$ Red arrowheads indicate T3 foci. $(G)$ Red arrows indicate sites where foci (12 of 23) are located near an SC twist. $(H)$ Turning the nucleus allows us to see that T3 foci are partially above SCs (red arrowhead). $\mathrm{T} 1$ foci are missing because they fade during processing. $(I, I)$ Late pachytene. (I) T3 foci (red arrowheads) diminish in number, but T1 foci (white arrows) remain. (J) Merge with Sme4 GFP. $(K)$ Early diffuse stage. Most persisting T3 foci (red arrowheads) remain associated at the end of an SC stretch (marked by Sme4-GFP). $(L)$ Diplotene. The seven bivalents (DAPI, in red) show at least one Hei10-GFP focus (red arrowheads). Bars, $2 \mu \mathrm{m}$. See also Supplemental Figure S3, A-I, for staging of pachytene nuclei and details for diffuse and diplotene nuclei (Supplemental Fig. S3J-O).

both Heil0 and Msh4. T3/Msh4 cofoci then lose their Msh4 but retain their Hei10, with T3 foci persisting through diplotene.

\section{Mid-late pachytene Hei10 T3 foci correspond in number and localization to CO-related "late" recombination nodules (LNs) and chiasmata}

T3 foci mark the sites of CO-fated recombinational interactions by several types of evidence.

First, their number (22 plus or minus three) (see above) corresponds exactly to the number of chiasmata seen at diplotene (21 plus or minus three; $n=100$ nuclei). Furthermore, 19-23 T3 foci $(n=20)$ persist at diplotene, where they are seen at chiasma sites, thus directly correlating T3 foci to sites of $\mathrm{CO}$ recombination (Fig. 2L; Supplemental Fig. S3M-O).

Second, ultrastructural analysis in several organisms, including Sordaria, has shown that sites of COs as defined genetically and/or by chiasmata are marked by LNs along the SCs (e.g., Carpenter 1979; Zickler et al. 1992). In Sordaria, the number of T3 foci (22 plus or minus three) is the same as the number of the LNs seen in electron microscopy (EM; 20-24 in 15 serially sectioned pachytene nuclei) (Zickler et al. 1992; D Zickler, unpubl.). This correspondence is seen also on a per bivalent basis. For example, bivalent 1 (longest) and bivalent 7 (smallest) exhibit, respectively, $4.2 \pm 0.9$ and $2.7 \pm 0.5 \mathrm{~T} 3$ foci compared with four to five and two to three LNs.

Third, the distribution of the T3 foci exhibits the two major hallmarks of meiotic COs: interference and the presence of the "obligatory CO" (Berchowitz and Copenhaver 2010). Moreover, there is a quantitative match between distribution of $\mathrm{T} 3$ foci and the distribution of LNs.

(1) CO interference is classically defined by coefficient of coincidence (CoC) analysis. Chromosomes are divided into intervals, and for all pairs of intervals along a chromosome, the probability that a bivalent will actually exhibit COs in both intervals is compared

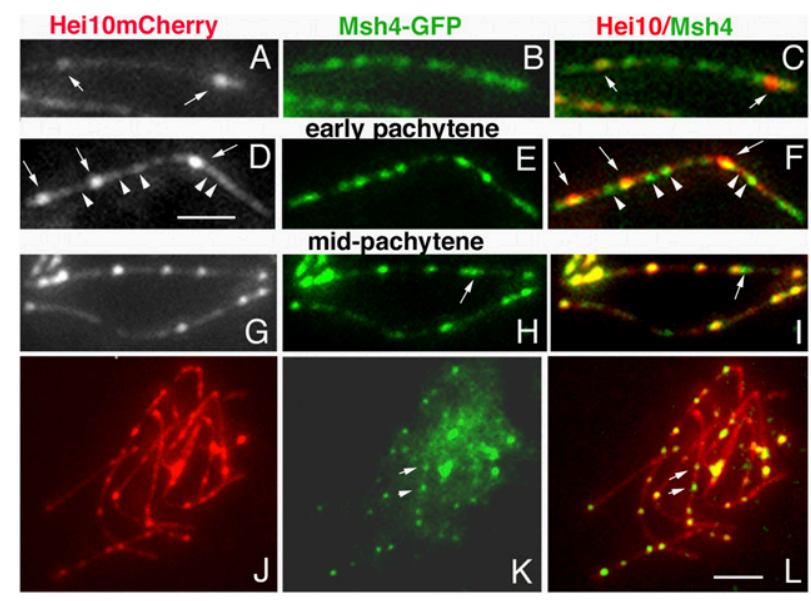

Figure 3. Colocalization of Heil0-mCherry and Msh4-GFP foci. At late zygotene $(A-C)$ and early pachytene $(D-F)$, only a subset of Msh4 foci (two and three in these magnified views) colocalize with T2 foci (arrows in $A, C, D, F)$. Most of the other Msh4 foci $(B, E)$ colocalize with T1 foci (arrowheads in $D, F)$. $(G-L)$ At mid-pachytene, almost all Msh4 foci colocalize with a Heilo focus (arrows in $H, I, K, L$ point to exceptions). $(G-I)$ Magnified view of two bivalents. Bars: $L, 2 \mu \mathrm{m} ; D, 300 \mathrm{~nm}$. 
with the probability expected from the frequencies of COs in the two intervals considered independently ("observed double COs/expected double COs"). A CoC curve gives the CoC (observed/expected) as a function of the distance between the two intervals that are being compared (the interinterval distance). For a given CoC curve, $\mathrm{L}_{\mathrm{CoC}}$ is the interinterval distance (on the $X$-axis) at which observed/expected is 0.5 (Zhang et al. 2014). Measurements of T3 positions along 658 bivalents from 120 mid-pachytene nuclei show that the T3 foci exhibit a CoC pattern reflecting interference: Double COs are very rare for small interinterval distances and increase in frequency as interinterval distance increases, similar to short (Fig. 4A, gray) and long (Fig. 4A, red) chromosomes (Fig. 4A, left). For T3 foci, CoC $=0.5$ when the distance between the centers of the two compared intervals is $1.3 \mu \mathrm{m}\left(\mathrm{L}_{\mathrm{CoC}}=1.3 \mu \mathrm{m}\right)$ (Fig. 4A). Interference and a tendency for even spacing are also indicated by $\gamma$ distribution analyses, with values of the parameter $\boldsymbol{v}$ of $6.77 \pm 0.49$ for the long chromosomes and $5 \pm 0.24$ for the smallest chromosomes. LNs exhibit the same degree of interference as T3 foci by both criteria, as illustrated for the two longest bivalents (most easily identified in all serially sectioned nuclei). CoC curves for LNs are virtually overlapping with those of $\mathrm{T} 3$ foci, with $\mathrm{L}_{\mathrm{CoC}}=1.3$ $\mu \mathrm{m}$ in both cases and very similar corresponding distribution of foci/LNs per bivalent (Fig. 4B). T3 foci and LNs also exhibit similar $\gamma$ parameter values: $\boldsymbol{v}=$ $8.5 \pm 1.1$ for LNs versus $6.77 \pm 0.49$ for T3 foci.

(2) In most organisms, bivalents acquire at least one CO, as required for homolog segregation, even when the average number of COs per bivalent is very low (Jones and Franklin 2006). Among 100 wild-type diplotene nuclei analyzed, all exhibit at least one chiasma per bivalent, and all 658 mid-pachytene bivalents exhibit at least one T3 focus $[P(0) \leq 0.002]$ when, based on Poisson distribution, $\sim 35$ zero-focus bivalents are expected.

These findings imply that there is a one-to-one-to-one relationship among diplotene chiasmata, pachytene LNs, and Hei10 T3 foci. In accord with this conclusion, studies in Arabidopsis and mice have shown colocalization of Hei10 with Mlh1 foci, which are also correlated with LNs and/or chiasmata (Lhuissier et al. 2007; Chelysheva et al. 2012; Qiao et al. 2014). Although we do not have a cytological marker for Sordaria Mlh1 (despite considerable effort), we infer that Sordaria T3 complexes correspond to those previously described in Arabidopsis and mice.

Hei10 T2 foci correspond to total EM-defined recombination nodules (LNs plus early nodules [ENS])

The observations presented above suggest that the $\sim 40$ Heilo T2 foci mark a specific subset of recombinational interactions that is intermediate in number between total interactions indicated by Mer3 and Msh4 foci $(\sim 80)$ and the number of COs/chiasmata ( 22). Additional evidence
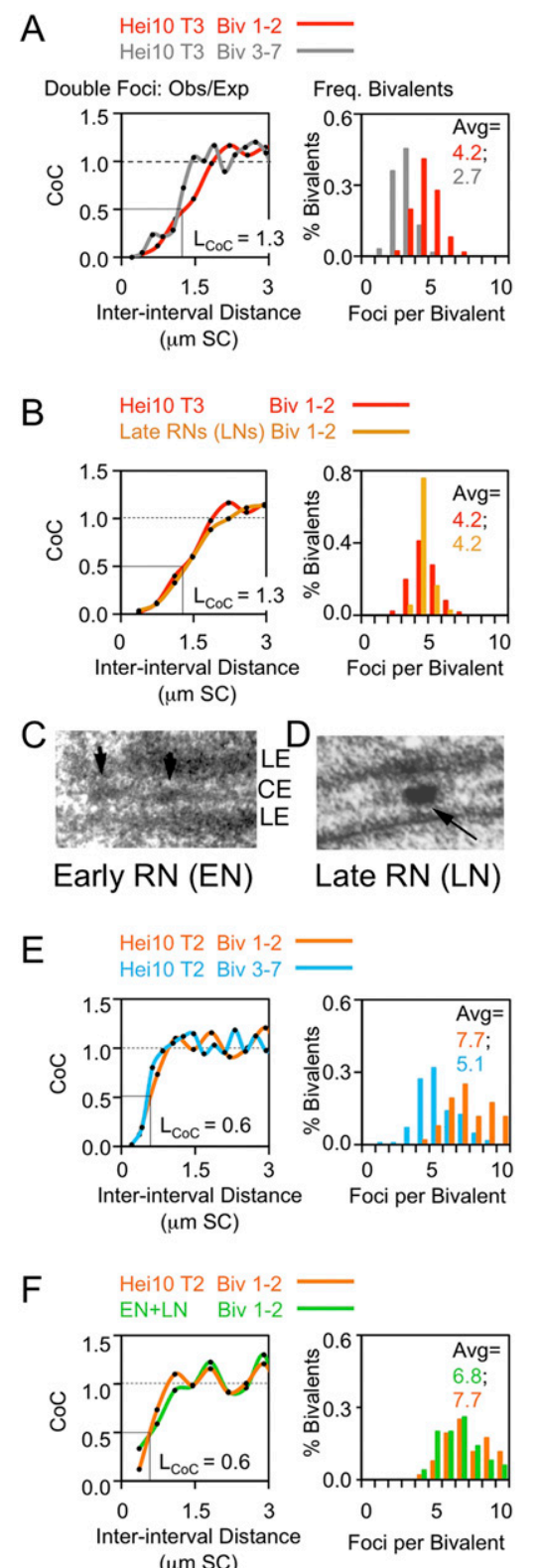

Figure 4. $\mathrm{CO}$ interference and number plus distribution of $\mathrm{T} 2$ and T3 foci, LNs, and ENs. $(A, B, E, F$, left $)$ CoC curves. (Right) Numbers and distribution of foci/nodules. $(A)$ T3 foci along long (red) and short (gray) bivalents. (B) T3 foci (red) and LNs (orange) along long and short bivalents. $(C, D)$ EM pictures of ENs (arrows; left) and LNs (arrow; right). (LE) Lateral element; (CE) central element. (E) T2 foci along long (orange) and short (blue) bivalents. (F) T2 foci (orange) and ENs + LNs (green) along long and short bivalents. Sample sizes: T3 in 188 long (biv 1-2) and 470 short (biv 3-7) bivalents (94 nuclei); T2 in 50 long and 125 short bivalents (25 nuclei); LNs and ENs along 40 long bivalents.

regarding the nature of $\mathrm{T} 2$ interactions is provided by further consideration of EM data. Ultrastructural analysis identifies a second type of SC-associated nodule, called "early nodules" (ENs). ENs (Fig. 4C) are usually smaller than LNs (Fig. 4D) and disappear at the transition from early to mid-pachytene, while LNs persist into late pachy- 
tene and diplotene (for review, see von Wettstein et al. 1984; Zickler and Kleckner 1999). The function of ENs is not established, although it was assumed that they reflect recombinational interactions that mature to fates other than COs (Carpenter 1987).

Three lines of evidence suggest that Heilo T2/Msh4 cofoci correspond to the sum of LNs plus ENs. (1) Analysis of Sordaria serially sectioned 60 zygotene and 15 pachytene nuclei shows that early pachytene bivalents $(n=105)$ exhibit 18-25 ENs. When added to the 22-24 LNs, the total number of nodules per nucleus is $40-50$. This number is the same as the number $(40.1 \pm 8.3)$ of early pachytene $\mathrm{T} 2$ foci (see above). (2) ENs emerge at zygotene and remain present through early pachytene before disappearing at the transition to mid-pachytene, exactly like T2/Msh4 cofoci. (3) T2 foci and LNs + ENs occur with the same distribution along the bivalents. By CoC analysis, T2 foci exhibit interference: $\mathrm{L}_{\mathrm{CoC}}=0.6 \mu \mathrm{m}$ for both long and short bivalents (Fig. 4E). Similarly, interference and a tendency for even spacing is indicated by $\gamma$ values of $\boldsymbol{v}=4.33 \pm$ 0.32 and $4.64 \pm 0.27$ on long and short chromosomes, respectively. Moreover, as shown by analysis of the two longest bivalents, CoC relationships for ENs + LNs are indistinguishable from that for $\mathrm{T} 2$ foci, again with $\mathrm{L}_{\mathrm{CoC}}=$ $0.6 \mu \mathrm{m}$ (Fig. 4F), and the two groups also exhibit very similar values of the $\gamma$ parameter $(\boldsymbol{v}=3.2 \pm 0.24$ for total nodules vs. $4.66 \pm 0.27$ for $\mathrm{T} 2$ foci).

\section{Heilo is not required for early events}

of recombination, for homolog coalignment, or for SC formation but is required for emergence and normal morphology of Msh4 foci

Previous analyses of events in wild-type meiosis (Storlazzi et al. 2010; Espagne et al. 2011) showed that robust chromosome axes develop at early leptotene with accompanying initiation of recombination as marked by foci of the RecA homolog Rad51. These double-strand breaks (DSBs) then allow recognition and pairing of homologs, with axis coalignment mediated by post-DSB helicase Mer3 and Msh4. Synapsis then occurs, bringing homolog axes into 100-nm juxtaposition along their lengths via formation of the SC.

Analysis of these events in a hei10D-null mutant (see the Supplemental Material for details) reveals normal axis morphogenesis, DSB-mediated coalignment, and SC morphogenesis. (1) In hei10D, as in wild type, Spo76/Pds5 and cohesin Rec8 load at $\mathrm{S}$ phase and form complete lines along all chromosomes from early leptotene to end pachytene (Fig. 5A-D). (2) As in wild type, hei10D chromosomes align synchronously (Fig. 5A,B) and then progress normally to the synapsis distance of $100-110 \mathrm{~nm}$ (Fig. 5C,D), with the seven pairs progressing synchronously (Fig. 5A-D). (3) Correspondingly, SC central region component Sme4 localizes between chromosomes in stage-specific correlation with synapsis to form smooth bright lines along all homologs at pachytene (Fig. 5E,F).

With respect to recombination, the hei10D mutant also exhibits normal numbers of Rad51 and Mer3 foci, with numbers per nucleus of 49 plus or minus seven and

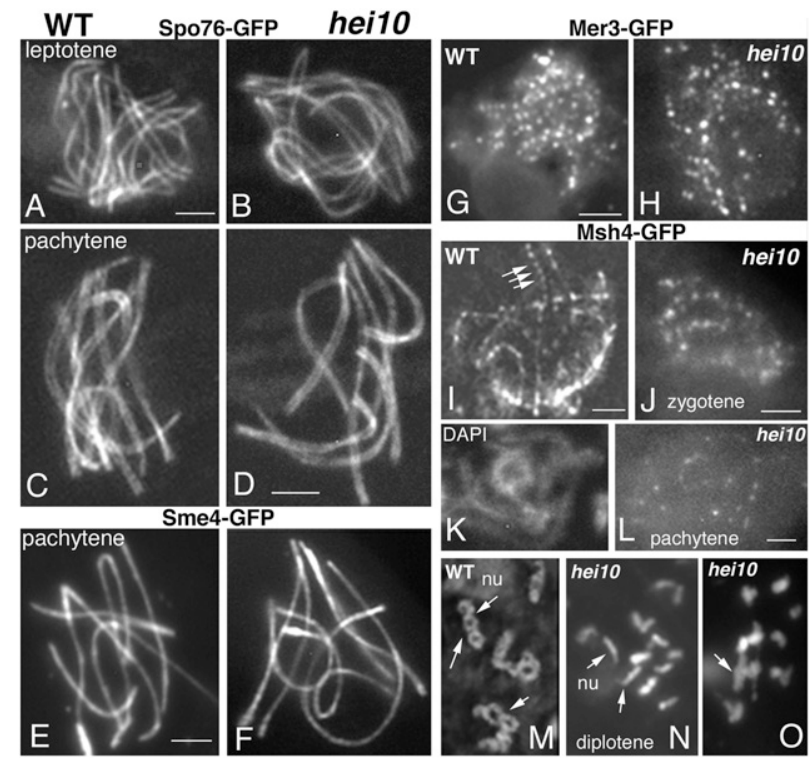

Figure 5. Prophase phenotypes of hei10D versus wild type (WT). $(A-D)$ Chromosome axes are stained by Spo76-GFP. Similar alignment and synapsis in wild type and hei10 at leptotene $(A, B)$ and pachytene $(C, D) .(E, F)$ At pachytene, Sme4-GFP makes seven smooth lines in wild type $(E)$ and hei10 $(F) .(G, H)$ Mer3 foci in wild type $(G)$ and hei10 $(H)$. (I-L) Msh4-GFP. (I) Wild-type early pachytene with evenly spaced foci (arrows). $(J-L)$ hei10. Foci are fewer and more irregular in shape and brightness at zygotene $(J)$ and pachytene $(L)(K$ shows corresponding DAPI). $(M-O)$ Diplotene nuclei. $(M)$ Wild type with several chiasmata (arrows) per bivalent. $(N, O)$ hei10 with either only univalents $(N)$ or few chiasmata (arrow in O). Bivalent 2 (attached to the nucleolus [nu]) shows no chiasma in hei10 (arrows in $N$ ) and four chiasmata in wild type (top arrows in $M$ ). Bars, $2 \mu \mathrm{m}$.

$105 \pm 15(n=90)$, respectively, as compared with 52 plus or minus eight and $135 \pm 10$ in wild type /representing $\sim 75$ evenly spaced matched pairs of Mer3 foci as in wild type; $n=100$ ). Also as in wild type (Fig. 5G), in hei10D, Mer3 foci are regularly spaced (Fig. $5 \mathrm{H}$ ) and move from an on-axis position to a between-axis position concomitant with SC formation before disappearing at early-mid pachytene as in wild type. However, in striking contrast, Hei10 is essential for normal appearance and morphogenesis of the Msh4 foci at and after zygotene. While wild-type nuclei exhibit $\sim 80$ regularly spaced Msh4 foci at late zygotene/early pachytene (81 plus or minus seven) (Fig. 5I), hei10D exhibits a reduced number of Msh4 foci, 25 plus or minus eight foci at mid-late zygotene (Fig. 5J), followed by an even more drastic reduction, with only eight to 15 foci per nucleus (seven plus or minus four) at early pachytene (Fig. $5 \mathrm{~K}, \mathrm{~L})$ and none thereafter $(n=50$ nuclei for each stage). Moreover, most of those Msh4 foci are fainter and less regular in shape in hei10D than in wild type (Fig. 5, cf. L and I).

These results indicate also that the formation of a subset of the $\sim 80$ Msh4 foci seen in wild type is Heiloindependent. The maximum number of Msh4 foci observed in hei10D $(\sim 25)$ and the timing of the disappearance of those foci (early pachytene) are very similar to the 
number and timing of the disappearance of the Msh4 foci that normally lack a colocalizing T2 focus. This relationship suggests that Heilo is required for assembly or stabilization of Msh4 at the subset of recombinational interactions that also develop Hei10 T2 foci (see above).

In accord with defective development of Msh4 complexes, hei10D exhibits a catastrophic reduction in $\mathrm{CO} /$ chiasma formation. Wild-type diplotene nuclei exhibit 21 plus or minus three chiasmata $(n=100)$ (Fig. $5 \mathrm{M})$. In hei10D, there is a $90 \%$ reduction in the total frequency of chiasmata: Thirty percent of the 30 analyzed diplotene nuclei contained only univalents (Fig. 5N), implying no mature COs, and $70 \%$ contained one to five bivalents, with only one chiasma per bivalent (arrow in Fig. 5O) versus two to four per bivalent in wild type (arrows in Fig. $5 \mathrm{M})$. There is no evidence of chromosome fragmentation in hei10D. Thus, DSBs are efficiently repaired as non-COs (NCOs) and/or by intersister interactions. However, progression of meiosis is perturbed: Prophase is prolonged by $>14 \mathrm{~h}$ (24-26 instead of $12 \mathrm{~h}$ in wild type), and this delay is dependent on Spol1-initated DSB formation (12-14 h in the spo11D hei10D double mutant).

SC formation being coupled to recombination in Sordaria, aberrant Msh4 dynamics in hei10D raises the possibility that hei10D is normal for SC formation because it disrupts the recombination-SC formation coupling, permitting SC formation irrespective of Msh4. This is not the case: The hei10D msh4D double mutant shows the same extensive defects in both recombination and synapsis as the single msh $4 D$ mutant, reflecting SC/recombination coupling (data not shown). Thus, Heilo is not required for SC formation but dramatically affects recombination complexes that are evolving in the context of forming SCs.

\section{Mutation of Hei10's RING domain confers a hei10-null phenotype}

HEI10 proteins contain a conserved C3H2C3-type RING domain (RFM) known to specify the catalytic activities of E3 ubiquitin ligases (Deshaies and Joazeiro 2009; Budhidarmo et al. 2012), and human HEI10 has an E3 ubiquitin ligase activity (Toby et al. 2003). To test the role of the RFM domain in Sordaria Hei10, we made a sitedirected mutation in this motif $\left(\right.$ hei $\left.10^{\mathrm{H} 30 \mathrm{~A}}\right)$ that is predicted to abolish E3 ligase activity (Supplemental Fig. S1; Supplemental Material). Phenotypes of hei10 $\mathrm{H} 30 \mathrm{~A} /$ hei10D strains are indistinguishable from those of hei10D: (1) only $5 \%-6 \%$ of eight-spored asci (Supplemental Fig. S4A,B) when compared with the $100 \%$ seen in wild type $(n=$ 400 asci) (Supplemental Fig. S4C); (2) similarly highly reduced levels of chiasmata, which occur at $9 \%$ the wildtype level $(n=20)$ (Fig. 6A); but (3) with normal SC formation (Fig. 6B). Thus, the RING motif and, by extension, its E3 ligase activity are required for Heil0mediated $\mathrm{CO} /$ chiasma formation.

We also examined the ability of the hei10 ${ }^{\mathrm{H} 30 \mathrm{~A}}$-GFP fusion protein to form foci. The mutant protein is no longer seen during meiotic prophase (Fig. 6C,D), implying no nucleation of $\mathrm{T} 1$ foci along the $\mathrm{SC}$ and no amplification
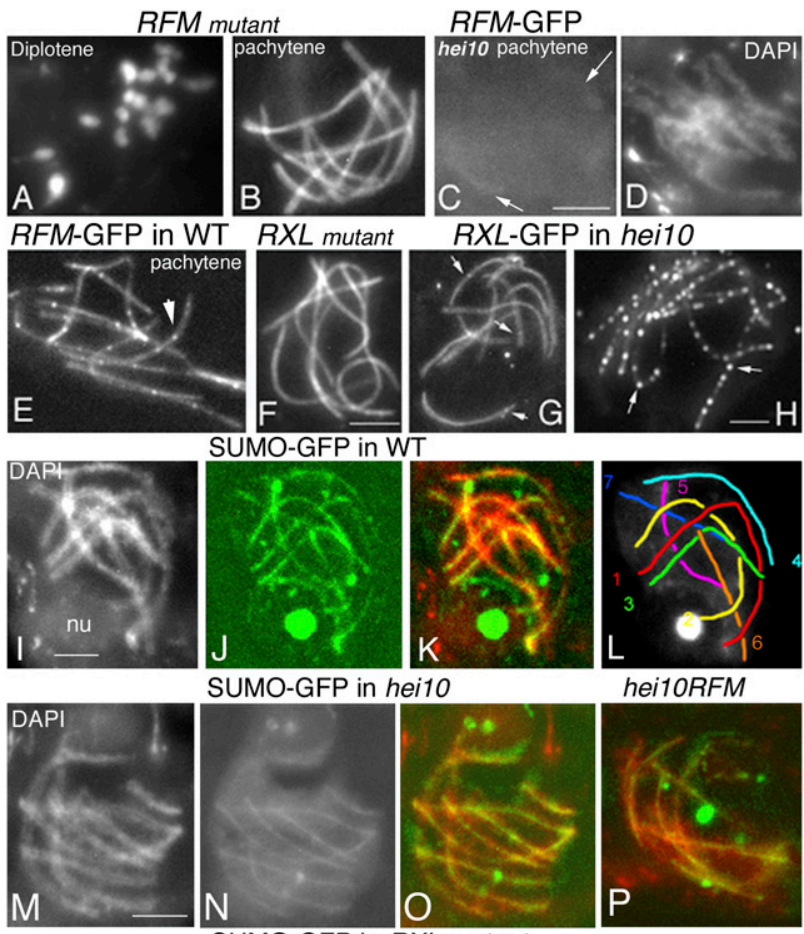

SUMO-GFP in hei10
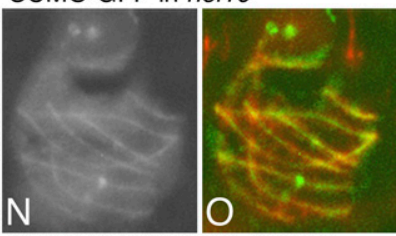

hei10RFM

SUMO-GFP in RXL mutant
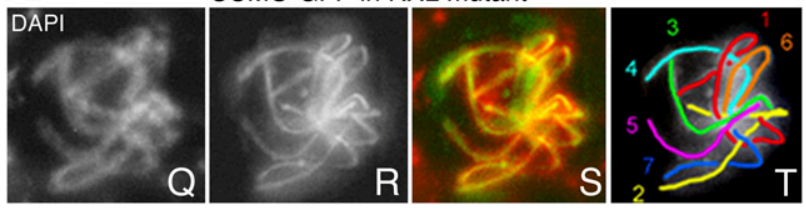

Figure 6. Phenotypes of hei10 mutants and SUMO-GFP in wild type (WT) and hei10 mutants. $(A, B)$ RFM mutant. (A) Diplotene with 14 univalents (DAPI). (B) Normal synapsis at pachytene (Spo76-GFP staining). (C,D) RFM-GFP in hei10D: No foci are formed $(C)$, corresponding to DAPI $(D)$, but the perinuclear area is stained (arrows in $C$, below). (E) T3 foci of RFM-GFP (arrow) in wild type (costained by Spo76-GFP). (F) RXL mutant. Sme4-GFP indicates normal SC formation. $(G, H)$ RXL-GFP in hei10D. $(G)$ Nucleus with only few foci (arrows; Spo76-GFP costaining). (H) Nucleus with numerous T2/T3 foci (arrows). (I-T) SUMOGFP localization at pachytene. Quantitative comparisons of SUMO intensities in wild-type and mutant strains in Supplemental Figure S5. (I-L) Wild type. (I) DAPI. (J) SUMO-GFP. $(K)$ Merge. ( $L$ ) Cartoon of the seven bivalents. The bright large spot corresponds to the nucleolus (nu in I). (M-O) hei10D. (M) DAPI. (N) SUMO-GFP. (O) Merge. (P) RFM mutant; merge with DAPI (red). (Q-T) SUMO-GFP accumulates in the RXL mutant. (Q) DAPI. (R) SUMO-GFP. (S) Merge. (T) Corresponding cartoon. Bars, $2 \mu \mathrm{m}$

of those foci into T2 and T3 foci at colocalizing subsets of recombination interactions. Failure of hei10 ${ }^{\mathrm{H} 30 \mathrm{~A}}$-GFP to localize to the SC is not a function of the GFP tag. Hei10GFP is fully functional (controls in the Supplemental Material). Moreover, in a background where wild-type Heilo is still present, hei10 ${ }^{\mathrm{H} 30 \mathrm{~A}}$-GFP shows the same dynamic localization along the SC as Hei10-GFP (cf. Figs. $6 \mathrm{E}$ and $2 \mathrm{E})$. Thus, the mutant protein, even with its GFP tag, is recessive, and the absence of foci in hei10D is a reflection of its null mutant phenotype. This finding also 
suggests that the wild-type protein can recruit the mutant protein to the chromosomes, implying the existence of protein-protein interactions as expected for assembly of the several types of Heilo foci. Thus, multimerization of Heilo into foci requires Hei10 E3 ligase activity. Heil0 focus formation could be autocatalytic, initially giving small $\mathrm{T} 1$ complexes and then, in response to the presence of an appropriate recombinosome, self-amplifying into T2 and T3 complexes.

\section{Hei10's RXL domain is required to elicit T1 foci} and constrain Heilo focus amplification

The Sordaria Heilo protein sequence (Supplemental Fig. S1) contains both an RXL consensus sequence, which is supposed to serve as docking site for the binding of cyclin-CDKs, and putative CDK phosphorylation sites (Ward et al. 2007). To test the role of the RXL motif, we introduced two point mutations in the most conserved region of this domain (Supplemental Fig. S1; Supplemental Material).

The $R X L$ hei10 ${ }^{\mathrm{R} 93 \mathrm{~A}, \mathrm{~L} 95 \mathrm{~A}}$ mutant has no defect in pairing or SC formation as judged by normal Sme4 localization (Fig. 6F), in accord with the absence of a role for Heil0 in these processes (see above). However, the mutant exhibits defects in both recombination and sporulation. The frequency of chiasmata is reduced by $77 \%$; thus, less than in the null or RFM mutants (see above): Among $30 R X L$ diplotenes, three showed seven bivalents with one chiasma per bivalent (compared with two to four per bivalent in wild type), 27 contained two to six bivalents with one chiasma each, and none showed only univalents (vs. 30\% in hei10D) (see above). In addition, the RXL mutant exhibits $30 \%$ of eight-spored asci (Supplemental Fig. S4D; $n=$ 400 ) versus $5 \%$ in hei10D and the RFM mutant and 100\% in wild type (Supplemental Fig. S4A-C).

Localization of the mutant protein $R X L$-GFP in a hei10D background is clearly defective but in an interesting way. First, 95\% of nuclei show either no foci or one to four faint T3-like foci in $20 \%$ of those nuclei $(n=100)$ (arrows in Fig. 6G). These foci might nevertheless be functional, thus explaining the increase of chiasmata /compared with hei10D) (see above). Second, the remaining 5\% of nuclei exhibit the wild-type number of $\mathrm{T} 1$ foci at zygotene, and then, by mid-pachytene, all of these foci now become brighter, with morphologies similar to T2-T3 foci (Fig. $6 \mathrm{H})$ in a total number (105-110) corresponding to the total number of Heilo foci. Those foci persist through late pachytene. Thus, in these nuclei, all $\mathrm{T} 1$ foci are amplified to a T2-T3 form irrespective of the presence or absence of an associated recombination complex (only eight plus or minus four Msh4 foci at pachytene in the mutant). This minority phenotype, never seen in the null or RFM mutants, suggests that hei10 ${ }^{\mathrm{R} 93 \mathrm{~A}}, \mathrm{~L} 95 \mathrm{~A}$ is not a simple hypomorph but rather is qualitatively altered in its functionality. Also interestingly, prophase is delayed by $>20 \mathrm{~h}$ in hei10 ${ }^{\mathrm{R} 93 \mathrm{~A}, \text { L95A }}$, more than in hei10D (14 h), again pointing to a nonhypomorphic condition.

Assignment of specific cyclin-binding function to an RXL motif is somewhat more problematic than assign- ment of E3 ligase function to an RFM motif (e.g., Ding et al. 2013). Nonetheless, the above phenotypes are consistent with a role for cyclin binding in the efficiency, timing, quality, and specificity with which Heilo is recruited to sites of recombination interactions and, additionally, with the ability of the recruited Heilo to disappear with appropriate timing (Discussion).

\section{Hei10 positively and negatively modulates SUMO localization along SCs by its RING and RXL domains, respectively}

Sordaria Heilo shows two putative SUMO-interacting motifs (Supplemental Fig. S1). SUMOylation is a prominent feature of meiotic chromosomes. SUMO occurs along the SCs in budding yeast and humans, suggesting a role in SC formation (Cheng et al. 2006; Hooker and Roeder 2006; Brown et al. 2008; Klug et al. 2013; VoelkelMeiman et al. 2013). We therefore investigated whether Hei10 plays any role for SUMO localization during Sordaria meiotic prophase.

Sordaria's single SUMO-encoding gene was tagged by GFP at its $\mathrm{N}$ terminus with no effect on normal function (Supplemental Material). Sordaria SUMO-GFP displays a diffuse staining in all nuclei of both vegetative and sexual cycles. In addition, during meiotic prophase, SUMO-GFP localizes to the SC central region, indicating that the SC proteins are SUMOylated. SUMO signal is first detected at zygotene as dotted linear structures along forming stretches of SCs. At pachytene, SUMO lines extend from telomere to telomere along each of the seven bivalents, and double staining with DAPI indicates that the SUMO lines are located between the synapsed homologs (Fig. 6IL). SUMO-GFP is still present after SC disassembly and during the diffuse stage as a cloud of dots and remains as a faint nuclear staining from diplotene to metaphase I (data not shown). SUMO localization along SCs is absent in sme4D, which does not form SCs, in agreement with the suggested role of SUMO in SC formation (e.g., Voelkel-Meiman et al. 2013). SUMOylation is not observed in prominent foci along the SCs (Fig. 6J) and thus does not detectably mark recombination complexes and/ or their associated T2/T3 foci.

Proper localization of SUMO along the SC does not depend on Hei10. SUMO-GFP localizes along the SC central region in hei10D like in wild type (Fig. 6M-O). However, SUMO lines are less bright in hei10D than in wild type, implying reduced SUMOylation of SC central components with accompanying increased nuclear background as compared with wild type (Fig. 6, cf. N and J). The same pattern is seen in the RFM mutant (Fig. 6P). In contrast, and oppositely, when the RXL domain is mutated, SUMO-GFP hyperaccumulates along the SC, as indicated by the dense bright lines of SUMO in the mutant (Fig. 6Q-T). Comparison of SUMO intensity in the different strains is shown in Supplemental Figure S5. These results indicate that Heilo is not the primary mediator of SC SUMOylation. Instead, Hei10 emerges as a modulator of the level of that SUMOylation, positively enhancing SUMOylation via its RING domain and, oppositely, acting 
negatively to moderate SUMOylation when its RXL domain is mutated.

Hei10 has additional roles during the sexual cycle at the nuclear periphery and for cytoskeleton-driven SPB dynamics

Nuclear periphery Heil0-GFP appears as a dense perinuclear halo from leptotene (Fig. 7A-C) through zygotene (Fig. 7D) and pachytene (Fig. 7E). This halo is meiotic prophase specific and is independent of chromosomal localization of Hei10: It is visible in the absence of the SC (sme4D) and DSBs (spo11D) and thus in the absence of recombination-related Heilo foci. The halo is also present in both non-null hei10 mutants. However, RXL-GFP (Fig. 7F) is brighter than RFM-GFP or Heil0-GFP staining, suggesting modulation of Heil0 accumulation by its RXL determinant. Interestingly, this perinuclear localization of Hei10 corresponds in localization and timing to previously described EM perinuclear "dense material" seen around prophase nuclei of Sordaria and Neurospora species. This material was suggested to comprise SPB (fungal equivalents of centrosomes) material in correlation with the fact that the SPB, while present during karyogamy and diplotene, is not visible from leptotene to pachytene (Thompson-Coffe and Zickler 1992).

Centrosome/SPB Like mammal centrosomes, fungal SPBs are required for spindle microtubule (MT) assembly. In addition, SPBs play specialized roles for sporulation: They mediate the assembly of an umbrella of astral MTs that surrounds the nucleus and delimits a spatial territory in

\section{perinuclear localization of Hei10-GFP in WT}
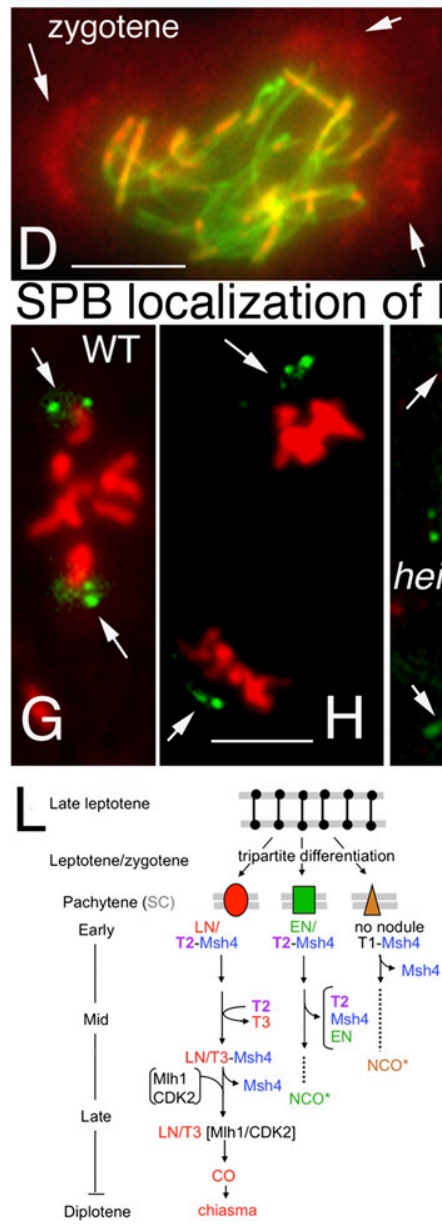
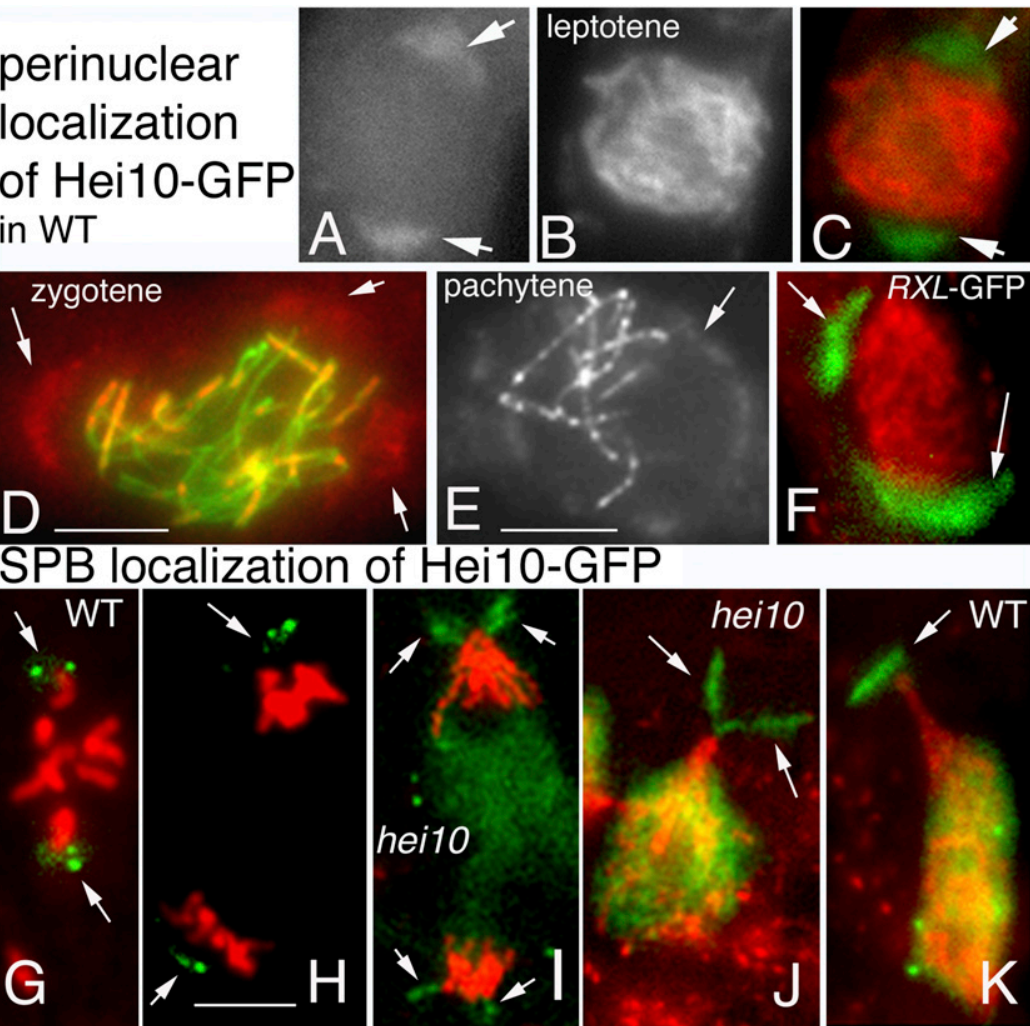

$\mathrm{M}$

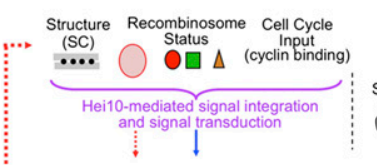

sumoylation
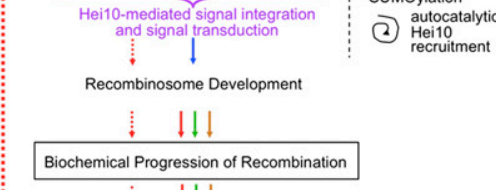

$$
\text { Bioche }
$$

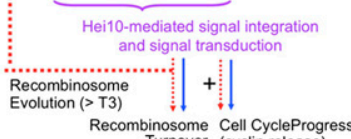

Recombinosome Cell CycleProgres
Turnover (cyclin release)
biquitination/STUbL (2) Hutocatalytic
Figure 7. Heilo perinuclear and SPB localization and a model of Heilo functions. $(A-E)$ Perinuclear Hei10-GFP in wild type (WT). $(A-C)$ At leptotene, Heil0-GFP forms two crescents (arrows). (B) Corresponding DAPI. (C) Merge. $(D)$ At zygotene, Hei10 enlarges (arrows); merge of Heilo-Cherry and Spo76-GFP. (E) At pachytene, the halo (arrow) surrounds the nucleus. $(F)$ $R X L$-GFP localization (leptotene) is similar but brighter (arrows). $(G-K)$ Heilo-GFP at SPBs merged with DAPI (red). $(G, H)$ Wild type. Heilo forms bright spots (arrows) at metaphase II $(G)$ and anaphase II $(H)$. $(I)$ hei10D anaphase II. Hei10 loads normally, but SPBs duplicate and enlarge precociously (arrows; cf. $H)$. $(J, K)$ Telophase II. hei10D: Duplicated SPBs $(J)$ have not separated (arrows), while at the same stage, wild-type SPBs $(K)$ are single (arrow). Bars, $2 \mu \mathrm{m}$. (L) Analysis of Sordaria Hei10 foci during meiotic prophase reveals that recombination complexes (starting from all recombination sites marked at leptotene by Mer3 foci that occur at matching sites, shown at the top) undergo tripartite morphological differentiation, shown as LN/T2/Msh4 (red ball), EN/T2/MSH4 (green rectangle), and no defined nodules/T1/Msh4 (brown triangle). (M) Model for Hei10 function as a structure-based signal transduction protein. Heilo integrates information from the SC (left), the state of the recombination complexes (middle; same symbols as in $L$ ), and the cell cycle. (Right) Heilo first promotes assembly via SUMOylation and then promotes disassembly by mediating turnover of those SUMOylated molecules in response to the progression of both the recombination complexes and the cell cycle (cyclin/CDK removal/inactivation) that licenses cell cycle progression to the next stage. 
the ascus (meiocyte) before ascospore enclosure. In accord with these specialized roles, SPBs undergo alterations in size, nucleating capacity, and orientation over the course of the sexual cycle (e.g., Espagne et al. 2011).

In wild-type cells, Hei10 localizes at SPBs once they reappear after pachytene through the two meiotic divisions and post-meiotic mitosis (Fig. 7G,H). Furthermore, Hei10 SPB localization is independent of its localization to the SCs (seen in sme4D and spo11D) and is present in both non-null hei10 mutants but with $R L X$-GFP brighter than RFM-GFP and wild-type staining, just as for perinuclear Heilo (above).

In correspondence with Hei10's SPB localization, all three hei10 mutants exhibit clear SPB defects: Duplication and separation of SPBs is no longer properly correlated with the divisions and spindle dynamics. The SPB duplicates precociously at the end of anaphase II (Fig. 7I). Consequently, telophase II nuclei show large duplicated SPBs (Fig. 7J) at a time when corresponding wild-type SPBs are single (Fig. $7 \mathrm{~K}$ ), and at following prometaphases, SPB separation is also delayed or partial. This separation defect is the same as that seen when wild-type meiocytes are treated by the anti-actin drug cytochalasinD (ThompsonCoffe and Zickler 1993), in accord with Heil0 localization to the nuclear envelope (NE)-associated cytoskeleton interface and implicating Heilo in actin-mediated SPB dynamics. Interestingly, these results also show that Hei10 localizes independently to the SCs and SPBs and that the RING and RXL domains are essential for SC/ recombinosome localization but not for SPB localization, implying a different substrate targeting mechanism.

\section{Discussion}

Our detailed analysis of Hei10's localization and roles in the Sordaria sexual cycle permits three general types of insights. First, the observed patterns suggest that Hei10 is a structure-based signal transduction protein that exerts its effects by a SUMO/ubiquitin "switch" (Fig. 7L,M). Second, integration of our findings with previous EM results reveals that meiotic prophase recombination complexes undergo tripartite morphological differentiation while concomitantly undergoing programmed differentiation at the DNA level into CO-fated and NCOfated types with accompanying $\mathrm{CO}$ interference. Third, Heilo is found to have broader roles in nonchromosomal aspects of the sexual cycle. These roles provide insight into related Heilo phenotypes described previously in nonmeiotic mammal cells.

\section{Hei10: a structure-based signal transduction protein}

The patterns described above suggest that Heilo is required for both development and turnover of specific subsets of recombination complexes (e.g., Msh4), first via T2 foci and then via T3 foci (Fig. 7L). Additionally, Hei10 specifically localizes to the SC central region (as T1 foci). Since recombination complexes (Mer3 and Msh4) localize to $\mathrm{T} 1$ foci prior to the evolution of T2 and T3 foci, Heilo exerts its effects specifically in the context of the SC. Thus, at each stage, Heilo would integrate informa- tion from underlying DNA recombination events and the SC to direct assembly of the appropriate recombination complexes in the appropriate structural context. Next, when recombination biochemistry has progressed appropriately, Heilo senses that change in state and switches functionality to mediate disassembly of the complex.

The role of Heilo in recombinosome turnover/evolution and CO/chiasma formation requires the RING (RFM) motif but also the putative cyclin-binding (RXL) motif, implying inputs from the cell cycle. Phenotypes of the hei10RXL mutant implicate cyclin as a positive factor for evolution of Heilo foci and thus recombination complexes. Additionally, this motif is implicated in selective targeting of Heilo focus amplification to specifically defined subsets of recombinational interactions and in turnover of recombination complexes (with a few nuclei exhibiting promiscuous, stable focus amplification). The latter phenotype is strikingly reminiscent of that seen for the mouse Hei10 ${ }^{\text {meit/meit }}$ mutant, which carries a partial deletion mutation in the cyclin-binding domain (Ward et al. 2007) and exhibits high levels of Msh4 foci until diplotene (Qiao et al. 2014).

Our analysis also suggests that, oppositely, Heil0 transduces information from the recombination process into the cell cycle. Cell cycle progression through prophase is delayed in the null and RFM mutants and is even further delayed in the $R X L$ mutant. Such delays are particularly notable because Sordaria lacks a robust "recombination/ SC checkpoint" mechanism (Storlazzi et al. 2010). These delays suggest that Heilo mediates communication between the state of Hei10 T2/T3-marked complexes such that progression of recombination tends to license cell cycle progression to later stages. In wild-type meiosis, the overall effect would be to promote nucleus-wide, synchronous progression of recombination in coordination with other (cell cycle-directed) events (Fig. 7M).

Taken together these effects imply that although Heilo is localized to the SC and is essential for normal recombination, it is neither an SC component nor a recombination protein per se. Instead, Hei10 is a structure-based signal transduction protein: It integrates information from a structural component (the SC), the state of the associated recombination complexes, and the cell cycle to direct formation and activation of those complexes in the appropriate place and time. Once the recombination process has appropriately progressed, Heilo senses this change and mediates evolution/turnover of the complexes to the next appropriate stage, concomitantly promoting the licensing cell cycle progression (Fig. 7M). This scenario could be generally applicable to other organisms. Most strikingly, in mice, the relationships between Msh4 and ZIP3/RNF212 and HEI10 (Reynolds et al. 2013; Qiao et al. 2014), taken together, are similar to those seen in Sordaria for Msh4 and Heilo.

\section{Hei10's interplay with SUMOylation and ubiquitination}

Absence of Hei10's RFM motif confers a hei10-null phenotype, directly implicating SUMO/ubiquitin E3 li- 
gase activity as a positive factor in Hei10-mediated recombinosome assembly. Although not required for general SUMOylation of the SC, Hei10 modulates SC SUMOylation in both directions, and this modulation can be correlated with variations in the level of Heilo itself. Diminution of SUMO in hei10D and hei10RFM is accompanied by the absence of Heilo foci. Enhanced SUMO in hei1ORXL is accompanied (in some nuclei) by promiscuous amplification of Heilo foci. These correlations suggest that amplification and loss of Heilo foci is accompanied by amplification and loss of SUMOylation.

SUMOylation is generally used to mediate the assembly of molecular complexes, whereas ubiquitination is used to mediate disassembly (for review, see Jentsch and Psakhye 2013). These two processes can be directly linked via the action of STUbL proteins, which directly target ubiquitination to SUMOylated molecules, thereby targeting them for proteasome degradation (for review, see Sriramachandran and Dohmen 2014). Given that Heil0 is involved in both the formation and turnover/disappearance of recombination complexes (see above), an attractive possibility is that Hei10 mediates a SUMO/ubiquitin switch-first promoting assembly via SUMOylation and then promoting disassembly by mediating turnover of those SUMOylated molecules (Fig. 7M). Related ideas have been described recently by Qiao et al. (2014), who propose that recombinosome differentiation (e.g., into $\mathrm{CO}$ and NCO types) involves a balance between opposing effects of SUMOylation and ubiquitination.

Tripartite differentiation of recombination complexes, including CO-designated complexes, during or before zygotene

It is widely accepted that, at the DNA level, a few DSBmediated interhomolog interactions are designated to be COs via an interference-mediated process, while the majority of remaining interactions mature as NCOs (Hunter 2006). In Sordaria, human oocytes, and tomatoes, LNs that correspond to the sites of CO-fated interactions are already present at zygotene and reach their full number by early pachytene with concomitant robust interference (Bojko 1985; Zickler et al. 1992; Lhuissier et al. 2007; see above). Thus, at least in these organisms, CO designation and accompanying interference appear to arise no later than zygotene. Similarly, in budding yeast, CO designation occurs at the leptotene/zygotene transition, as defined by the emergence of CO-specific intermediates at that stage and the concomitant emergence of CO-correlated Zip2/3 foci independent of the SC (Allers and Lichten 2001; Börner et al. 2004; Fung et al. 2004). Analogous timing has also recently been suggested for mice (Reynolds et al. 2013).

The above findings provide further information about events at zygotene. In Sordaria, as in all organisms studied so far (e.g., Zickler and Kleckner 1999), the total number of LNs + ENs $(\sim 45-50)$ is significantly less than the total number of DSB-initiated interhomolog interactions marked by Rad51, Mer3, and Msh4 foci ( 80) (Storlazzi et al. 2010). Thus, during zygotene, Sordaria exhibits three morphologically different types of recombination com- plexes: LNs, ENs, and non-nodule-associated interactions (Fig. 7L). Hei10/Msh4 focus morphologies and dynamics provide further evidence for such tripartite differentiation. Heilo is first enhanced at a subset of Msh4 foci, giving T2/Msh4 cofoci; the other Msh4 complexes remain T1-associated and disappear before mid-pachytene. T2/Msh4 cofoci then progress to two different fates. One subset, already fated to mature into COs/chiasmata, evolves into T3/Msh4 cofoci, also correlated with COfated LNs. The other T2/Msh4 subset, correlated with ENs in number, distribution, and disappearance, loses both Msh4 and Hei10 T2. T3/Msh4 foci then lose their Msh4 at late pachytene but remain as T3 foci up to diplotene, where they correspond in number and localization to chiasmata.

This cytologically tripartite differentiation appears to be superimposed on a simple duality of differentiation at the DNA level, thus raising several interesting questions. (1) In Sordaria, the ratio of CO-fated to NCO-fated interactions is about $1: 3$, as defined by genetic analysis (Zickler et al. 1992). If so, EN/T2-marked and non-nodule/ non-T2-marked interactions are fated for maturation into interhomolog NCOs despite their morphological differences. Do they also use the same biochemical pathway? (2) Why are there two morphologically different types of NCO-fated interactions, one of which (EN) is morphologically "more robust"? A possible hint comes from the fact that LNs and ENs are both closely associated with SC nucleation events (Zickler et al. 1992). Both types of structures might thus be robust because they play a role in SC nucleation. (3) What type of regulatory process can yield a total array of nodules (EN + LN) and T2/Msh4 cofocus complexes that exhibits interference as a group but contains embedded within it the classical set of designated highly interfering COs? Occurrence of pachytene complexes in a number intermediate between DSBs/total interactions and COs has led to the proposition that $\mathrm{CO}$ interference arises in two waves (de Boer et al. 2006; Yokoo et al. 2012; Libuda et al. 2013; Qiao et al. 2014). An interesting possibility to be explored elsewhere is that a single round of interference provides evenly spaced SC nucleations, with concomitant $\mathrm{CO}$ designation occurring as a programmed subroutine within this broader process.

\section{Hei10 has additional critical roles in SPB dynamics}

We discovered a completely new role for Heilo in SPB/ centrosome dynamics, beyond meiotic SC and recombination. Phenotypes of hei10 mutants implicate Heilo (and its RXL domain) in two aspects of SPB dynamics: (1) coordination of SPB duplication with cell cycle progression and (2) cytoskeleton-mediated SPB separation and movement along the nuclear periphery. In accord with these roles, Hei10 localizes at the relevant stages to the SPBs at the nucleus/cytoplasm interface but is not required for SPB morphogenesis. Moreover, Hei10 has distinct localizations and roles at the SPB and during prophase: Hei10 localization to the SPB is independent of its localization to the SC and of both the RING and RXL domains (in contrast to its SC localization). 
Interestingly, Sme4, like Hei10, also localizes to both the SC and the SPB. However, in both of its roles, Sme4 is a basic structural component: It mediates linkage of homolog axes via its role as an SC transverse filament protein and, at the SPB, change in the SPB morphology from a planar to a bent configuration (Espagne et al. 2011). In contrast, Hei10 localizes downstream from the formation of both the SC and the SPB and is not involved in their morphology. Moreover, from its SC location, Hei10 mediates the progression of the recombination complexes; analogously, from its SPB location, Hei10 coordinates SPB duplication and movement with cell cycle progression. Thus, for both the SPB and meiotic prophase recombination complexes (see above), Heilo is a transducer of information in events that involve prominent structures rather than being an intrinsic component of those structures per se.

\section{Implications for Hei10's roles in mammalian mitotic cells}

Previous studies have considered that Heilo might be important for cancer simply because it regulates (or deregulates) the cell cycle (e.g., Toby et al. 2003; Singh et al. 2007; Scoles 2008). The current findings, which implicate Heilo as an integrator and transducer of multiple signal inputs, significantly expand the range of possible roles of Heil0 in cancer. Also, Heilo's roles are specifically linked to Mlh1, with roles for base-pair mismatch correction, and to Msh4/5, which is in the same family as basic MutS homolog mismatch repair proteins (e.g., Msh2). Thus, in cancer cells, normal or aberrant expression of Hei10 could directly influence the repair process. Additionally, involvement of Heilo with the SPB raises the possibility that a primary target of Heil0-mediated effects for nonmeiotic cells, including cancer cells, could be perturbation of centrosome dynamics with resulting aneuploidy, a central factor in the emergence of the cancerous state. Perturbation of centrosome dynamics could also alter the cell cycle progression, including constraining of cancer cell proliferation (Singh et al. 2007; Scoles 2008).

\section{Materials and methods}

\section{Strains, cloning, and transformation}

S. macrospora wild type is St-Ismier FGSC 4818. Sordaria HEI10 (SMAC_03791) and SUMO (SMAC_08473) genes were identified from the Sordaria genomic DNA sequence (Nowrousian et al. 2010). The hei10D-null mutant was obtained by single-step gene replacement: A hygromycin resistance cassette replaces the entire ORF. The two point mutations hei10 ${ }^{\mathrm{H} 30 \mathrm{~A}}$ and hei10 ${ }^{\mathrm{R} 93 \mathrm{~A}, \mathrm{~L} 95 \mathrm{~A}}$ were created by PCR-based mutagenesis and introduced in wild type by cotransformation with a plasmid encoding the hygromycin resistance cassette. Additional information on plasmid construction, mutants, and GFP/mCherry tags is in the Supplemental Material.

\section{Cytology}

For cytological analysis, GFP, mCherry, and DAPI $(0.5 \mathrm{mg} / \mathrm{mL})$ signals were observed either on living material or after fixation in
4\% paraformaldehyde with a Zeiss Axioplan microscope with a CCD Princeton camera as described (Storlazzi et al. 2010) and with DeltaVision OMXTM platform (3D-SIM; Applied Precision). For both $\gamma$ and $\mathrm{CoC}$ analyses of interference, interfocus distances were measured from the center of the first focus to the center of the next focus using public domain software ImageJ (Supplemental Material).

\section{Acknowledgments}

We are extremely grateful to Edith Heard for providing access to her 3D-SIM microscope. We especially thank Esther de Boer for providing $\gamma$ distribution analyses. We acknowledge the excellent technical assistance of Christelle Vasnier and Sophie Tessé. This work, D.Z., E.E., and A.D.M. were supported by grants from the Centre National de la Recherche Scientifique (Unité Mixte de Recherche 8621) and the National Institutes of Health (GM044794 to N.K.). N.K. and L.Z. were supported by National Institutes of Health grant GM044794.

\section{References}

Allers T, Lichten M. 2001. Differential timing and control of noncrossover and crossover recombination during meiosis. Cell 106: 47-57.

Agarwal S, Roeder GS. 2000. Zip3 provides a link between recombination enzymes and synaptonemal complex proteins. Cell 102: 245-255.

Berchowitz LE, Copenhaver GP. 2010. Genetic interference: don't stand so close to me. Curr Genomics 11: 91-102.

Bhalla N, Wynne DI, Jantsch V, Dernburg AF. 2008. ZHP-3 acts at crossovers to couple meiotic recombination with synaptonemal complex disassembly and bivalent formation in $C$. elegans. PLoS Genet 4: e1000235.

Bojko M. 1985. Human meiosis. IX. Crossing over and chiasma formation in oocytes. Carlsberg Res Commun 50: 43-72.

Börner GV, Kleckner N, Hunter N. 2004. Crossover/noncrossover differentiation, synaptonemal complex formation, and regulatory surveillance at the leptotene/zygotene transition of meiosis. Cell 117: 29-45.

Brown PW, Hwang K, Schlegel PN, Morris PL. 2008. Small ubiquitin-related modifier (SUMO)-1, SUMO-2/3 and SUMOylation are involved with centromeric heterochromatin of chromosomes 9 and 1 and proteins of the synaptonemal complex during meiosis in men. Hum Reprod 23: 2850-2857.

Budhidarmo R, Nakatani Y, Day CL. 2012. RINGs hold the key to ubiquitin transfer. Trends Biochem Sci 37: 58-65.

Carpenter ATC. 1979. Synaptonemal complex and recombination nodules in recombination deficient mutants of Drosophila melanogaster. Chromosoma 75: 259-292.

Carpenter ATC. 1987. Gene conversion, recombination nodules, and the initiation of meiotic synapsis. Bioessays 6: 232-236.

Chelysheva L, Vezon D, Chambon A, Gendrot G, Pereira L, Lemhemdi A, Vrielynck N, Le Guin S, Novatchkova M, Grelon M. 2012. The Arabidopsis HEI10 is a new ZMM protein related to Zip3. PLoS Genet 8: e1002799.

Cheng CH, Lo YH, Liang SS, Ti SC, Lin FM, Yeh CH, Huang HY, Wang TF. 2006. SUMO modifications control assembly of synaptonemal complex and polycomplex in meiosis of Saccharomyces cerevisiae. Genes Dev 20: 2067-2081.

de Boer E, Stam P, Dietrich AJJ, Pastink A, Heyting C. 2006. Two levels of interference in mouse meiotic recombination. Proc Natl Acad Sci 103: 9607-9612.

Deshaies RJ, Joazeiro CA. 2009. Ring domain E3 ubiquitin ligases. Annu Rev Biochem 78: 399-434. 
Ding Q, Li L, Whyte P. 2013. Human papillomavirus 18 E1E4 protein interacts with cyclin A/CDK 2 through an RXL motif. Mol Cell Biochem 373: 29-40.

Espagne E, Vasnier C, Storlazzi A, Kleckner NE, Silar P, Zickler D, Malagnac F. 2011. Sme4 coiled-coil protein mediates synaptonemal complex assembly, recombinosome relocalization, and spindle pole body morphogenesis. Proc Natl Acad Sci 108: 10614-10619.

Fung JC, Rockmill B, Odell M, Roeder GS. 2004. Imposition of crossover interference through the nonrandom distribution of synapsis initiation complexes. Cell 116: 795-802.

Hooker GW, Roeder GS. 2006. A role for SUMO in meiotic chromosome synapsis. Curr Biol 16: 1238-1243.

Hunter N. 2006. Meiotic recombination. In Molecular genetics of recombination (ed. A Aguilera, R Rothstein), pp. 381-442. Springer Berlin/Heidelberg, Germany.

Jantsch V, Pasierbek P, Mueller MM, Schweizer D, Jantsch M, Loidl J. 2004. Targeted gene knockout reveals a role in meiotic recombination for ZHP-3, a Zip3-related protein in Caenorhabditis elegans. Mol Cell Biol 24: 7998-8006.

Jentsch S, Psakhye I. 2013. Control of nuclear activities by substrate-selective and protein-group SUMOylation. Annu Rev Genet 47: 167-186.

Jones GH, Franklin FCH. 2006. Meiotic crossing-over: obligation and interference. Cell 126: 246-248.

Klug H, Xaver M, Chaugule VK, Koidl S, Mittler G, Klein F, Pichler A. 2013. Ubc9 Sumoylation controls SUMO chain formation and meiotic synapsis in Saccharomyces cerevisiae. Mol Cell 50: 625-636.

Lhuissier FG, Offenberg HH, Wittich PE, Vischer NO, Heyting C. 2007. The mismatch repair protein MLH1 marks a subset of strongly interfering crossovers in tomato. Plant Cell 19: 862-876.

Libuda DE, Uzawa S, Meyer BJ, Villeneuve AM. 2013. Meiotic chromosome structures constrain and respond to designation of crossover sites. Nature 502: 703-706.

Luo Q, Tang D, Wang M, Luo W, Zhang L, Qin B, Shen Y, Wang K, Li Y, Cheng Z. 2013. The role of OsMSH5 in crossover formation during rice meiosis. Mol plant 6: 729-742.

Nowrousian M, Stajich JE, Chu M, Engh I, Espagne E, Halliday K, Kamerewerd J, Kempken F, Knab B, Kuo HC, et al. 2010. De novo assembly of a $40 \mathrm{Mb}$ eukaryotic genome from short sequence reads: Sordaria macrospora, a model organism for fungal morphogenesis. PLoS Genet 6: e1000891.

Qiao H, Prasada Rao HB, Yang Y, Fong JH, Cloutier JM, Deacon DC, Nagel KE, Swartz RK, Strong E, Holloway JK, et al. 2014. Antagonistic roles of ubiquitin ligase HEI10 and SUMO ligase RNF212 regulate meiotic recombination. Nat Genet 46: 194-199.

Reynolds A, Qiao H, Yang Y, Chen JK, Jackson N, Biswas K, Holloway JK, Baudat F, de Massy B, Wang J, et al. 2013. RNF212 is a dosage-sensitive regulator of crossing-over during mammalian meiosis. Nat Genet 45: 269-278.

Scoles DR. 2008. The merlin interacting proteins reveal multiple targets for NF2 therapy. Biochim Biophys Acta 1785: 3254.

Singh MK, Nicolas E, Gherraby W, Dadke D, Lessin S, Golemis EA. 2007. HEI10 negatively regulates cell invasion by inhibiting cyclin $\mathrm{B} / \mathrm{Cdk} 1$ and other promotility proteins. Oncogene 26: 4825-4832.

Snowden T, Acharya S, Butz C, Berardini M, Fishel R. 2004. hMSH4-hMSH5 recognizes Holliday junctions and forms a meiosis-specific sliding clamp that embraces homologous chromosomes. Mol Cell 15: 437-451.

Sriramachandran AM, Dohmen RJ. 2014. SUMO-targeted ubiquitin ligases. Biochim Biophys Acta 1843: 75-85.
Storlazzi A, Gargano S, Ruprich-Robert G, Falque M, David M, Kleckner N, Zickler D. 2010. Recombination proteins mediate meiotic spatial chromosome organization and pairing. Cell 141: 94-106.

Strong ER, Schimenti JC. 2010. Evidence implicating CCNB1IP1, a RING domain-containing protein required for meiotic crossing over in mice, as an E3 SUMO ligase. Genes 1: 440-451.

Thompson-Coffe C, Zickler D. 1992. Three microtubule-organizing centers are required for ascus growth and sporulation in the fungus Sordaria macrospora. Cell Motil Cytoskeleton 22: 257-273.

Thompson-Coffe C, Zickler D. 1993. Cytoskeleton interactions in the ascus development and sporulation of Sordaria macrospora. J Cell Sci 104: 883-898.

Toby GG, Gherraby W, Coleman TR, Golemis EA. 2003. A novel RING finger protein, human enhancer of invasion 10, alters mitotic progression through regulation of cyclin B levels. Mol Cell Biol 23: 2109-2122.

Voelkel-Meiman K, Taylor LF, Mukherjee P, Humphryes N, Tsubouchi H, Macqueen AJ. 2013. SUMO localizes to the central element of synaptonemal complex and is required for the full synapsis of meiotic chromosomes in budding yeast. PLoS Genet 9: e1003837.

von Wettstein D, Rasmussen SW, Holm PB. 1984. The synaptonemal complex in genetic segregation. Annu Rev Genet 18: 331-413.

Wang K, Wang M, Tang D, Shen Y, Miao C, Hu Q, Lu T, Cheng Z. 2012. The role of rice HEI10 in the formation of meiotic crossovers. PLoS Genet 8: e1002809.

Ward JO, Reinholdt LG, Motley WW, Niswander LM, Deacon DC, Griffin LB, Langlais KK, Backus VL, Schimenti KJ, O'Brien MJ, et al. 2007. Mutation in mouse hei10, an e3 ubiquitin ligase, disrupts meiotic crossing over. PLoS Genet 3: e139.

Yokoo R, Zawadzki KA, Nabeshima K, Drake M, Arur S, Villeneuve AM. 2012. COSA-1 Reveals robust homeostasis and separable licensing and reinforcement steps governing meiotic crossovers. Cell 149: 75-87.

Zhang L, Liang Z, Hutchinson J, Kleckner N. 2014. Crossover patterning by the beam-film model: analysis and implications. PLoS Genet 10: e1004042.

Zickler D, Kleckner N. 1999. Meiotic chromosomes: integrating structure and function. Annu Rev Genet 33: 603-754.

Zickler D, Moreau PI, Huynh AD, Slezec AM. 1992. Correlation between pairing initiation sites, recombination nodules and meiotic recombination in Sordaria macrospora. Genetics 132: $135-148$. 


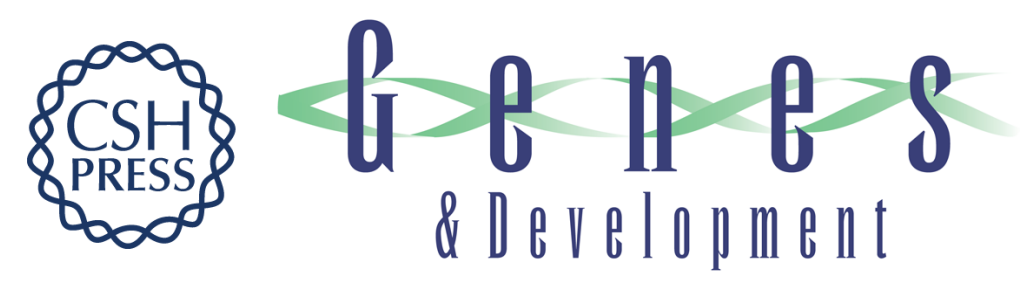

\section{E3 ligase Hei10: a multifaceted structure-based signaling molecule with roles within and beyond meiosis}

Arnaud De Muyt, Liangran Zhang, Tristan Piolot, et al.

Genes Dev. 2014, 28:

Access the most recent version at doi:10.1101/gad.240408.114

\section{Supplemental http://genesdev.cshlp.org/content/suppl/2014/05/14/28.10.1111.DC1 Material}

References This article cites 45 articles, 8 of which can be accessed free at: http://genesdev.cshlp.org/content/28/10/1111.full.html\#ref-list-1

Creative This article is distributed exclusively by Cold Spring Harbor Laboratory Press for the first Commons six months after the full-issue publication date (see

License http://genesdev.cshlp.org/site/misc/terms.xhtml). After six months, it is available under a Creative Commons License (Attribution-NonCommercial 4.0 International), as described at http://creativecommons.org/licenses/by-nc/4.0/.

Email Alerting Receive free email alerts when new articles cite this article - sign up in the box at the top Service right corner of the article or click here.

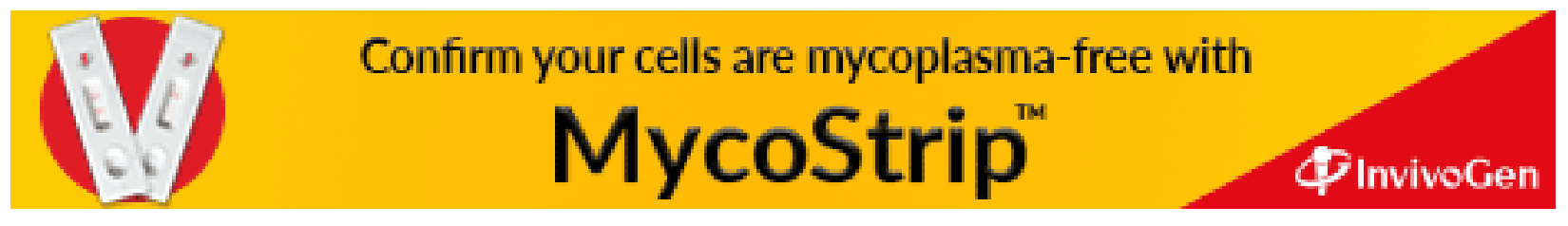

